\title{
LA METAHEURÍSTICA COLONIA DE HORMIGAS EN LA SOLUCIÓN DE UN PROBLEMA DE RUTAS. CASO PRÁCTICO DE ESTUDIO
}

\section{THE ANT COLONY METAHEURISTIC IN SOLVING A ROUTE PROBLEM. CASE STUDY}

iD Henrry Garrido A. ${ }^{1}$

(iD) Johnson Lomote V. ${ }^{1}$

${ }^{1}$ Universidad Nacional "Santiago Antúnez de Mayolo”, Huaraz. Perú

Correspondencia:

Mag. Henrry Garrido Angulo

hgarridoa@unasam.edu.pe

\section{RESUMEN}

El estudio de investigación está orientado a un problema de rutas sobre el recojo de visitantes instalados en hoteles de la ciudad de Huaraz desde un lugar de parqueo que serán trasladados a los destinos turísticos en la región de Ancash, mediante la utilización de la metaheurística colonia de hormigas, la cual es inspirada en el comportamiento de las hormigas y la emisión de la sustancia de feromona en la búsqueda de sus alimentos; permite la simulación de hormigas artificiales y el uso de la probabilidad, para diseñar un sistema de rutas de recorrido mínimo a través de la variable de longitud. Se realizó un estudio descriptivo no experimental y cuantitativo en base a 6 puntos-vértices, entre el lugar de parqueo y 5 hoteles. El análisis del estudio a un caso práctico, revela que considerando todos los puntos vértices iniciando y terminando en un mismo punto-vértice y en el marco teórico de la teoría del problema del agente viajero, se generaron 720 rutas, como posibilidades de estudio, y si se fijara un punto-vértice se generaron 120 rutas posibles, siendo así aun un estudio relativamente arduo; 46 rutas de este último caso resultarían ser todas diferentes. El resultado de la ruta de recorrido mínimo es la combinación 1234561 con $3909 \mathrm{~m}$.

Palabras clave: metaheurística; colonia de hormiga; probabilidad; ruta.

\section{ABSTRACT}

The research study is oriented to a routing problem on the collection of visitors installed in hotels in the city of Huaraz from a parking place, it will be transported to tourist destinations in the Ancash region using the metaheuristic ant colony inspired by the behavior of ants and the emission of the pheromone substance when they leave in search of their food, it allows to simulate with artificial ants and the probability, in order to design a minimum route system by means the length. A descriptive, non-experimental and quantitative study was carried out based on 6 vertex points, between the parking place and 5 hotels. The analysis of the study resulting from the application to a practical case reveals that considering all the vertex points starting 
and ending in the same vertex point and in the theoretical framework of the traveling salesman problem, 720 routes were generated, as possibilities study, and if a vertex point were established 120 possible routes were generated, even so, it is relatively arduous; 46 routes in the latter case would all turn out to be different. The result of the minimum travel route is the combination 1234561 with $3909 \mathrm{~m}$.

Keywords: metaheuristic; ant colony; probability; route.

\section{INTRODUCCIÓN}

Las metodologías de resolución de problemas en las ciencias constituyen herramientas valiosas, pues permiten resolver una parte y un aspecto de los problemas que se plantean en especial, en la Ciencia matemática. El estudio de investigación se orienta a otras metodologías denominadas metaheurísticas para resolver evidentemente problemas esencialmente en el campo de la optimización. Una de ellas, es el caso del algoritmo de colonia de hormigas (ACO), una metaheurística que representa a un fenómeno de comportamiento social de vida animal, es decir, de unos insectos conocidos como hormigas en el marco de un mecanismo natural de la ciencia biológica, motivo de inspiración, y en especial del comportamiento de las hormigas, con una significancia social especial, de su labor comunitario y colaborativo observando su manera de actuar de estos no siempre pequeños insectos; su sistema de convivencia y supervivencia resultan tener un comportamiento singular, y porque no decir de modelo y de lección de vida para el hombre.

Muchos de los problemas de optimización combinatoria pueden ser resueltos mediante los algoritmos de optimización colonia de hormigas desde el TSP, el problema de horarios y muchos problemas estocásticos. Una solución óptima muy próxima puede ser puede ser encontrado a través de ACO, pero todavía se está lejos de alcanzar soluciones exactas. Existe un cambio dinámico en la teoría de grafos en comparación de ACO y que pueden ser adaptados a cambios en tiempo real y correr continuamente. ACO es la técnica para encontrar el camino más corto para los problemas de ruta de vehículos; en un escenario real las hormigas encuentran la distancia más corta para ubicar el alimento mediante la feromona, sustancia química esparcida en el ruteo y que en destinos débiles dicha sustancia se evapora rápidamente, y que a su vez permite evaluar eficiencias (T Srinivas, 2018).

El mTSP Problema del Agente Viajero múltiple extensivo en la optimización combinatoria simplificados de los problemas de rutas de vehículos en el marco de los problemas NP duros. El ACO puede ser asignado al trabajo en equipos a través de una especie de misión que se hace inicialmente utilizando una estrategia max-min para trabajar juntos en la búsqueda de la solución óptima (Li-Chih y Tai-Wen, 2019).

El ACO multinivel en sus diversas aplicaciones resuelve problemas de planeamiento de transportes en actividades forestales. Los parámetros de ACO son automáticamente configurados al evaluar un parámetro de dominio combinado a través de varios niveles (Lin, Contreras, Dai y Zhang, 2016).

El ACO es una metaheurística de aproximación perteneciente a la familia de enjambres siendo un método que converge a las soluciones en un tiempo razonable, ACO puede ayudar a lograr pasos cómodos, sin embargo, ofrece el potencial para encontrar buenas soluciones a algunos problemas que podrían ser prohibidos por otros algoritmos en casos donde la función de costo debe ser dinámicamente calculados (Baeza, Ihle y Ortiz, 2017).

Debido a las restricciones de movimiento de los vehículos pesados, la mayoría de las ciudades han empezado a implementar centros receptores para reducir los impactos negativos ambientales, y para ello se ha desarrollado un modelo matemático para minimizar los costos totales de transporte incluyendo costos de rutas, costos de arreglos de vehículos, costos de emisión y de impuestos zonas de entrada (Juvvala y Sarmah, 2021). 
El ACO aplicado a los sistemas de administración de tráfico descentralizado que integra rutas de tráfico dinámico y de control de tráfico de intersección de señales libres para la conexión de vehículos. Cada conexión de vehículos se ha modelado como una hormiga, los depósitos de feromona en los caminos, mientras los sensores de rutas son agregados a la información de feromona creando condiciones de tráfico transfiriendo información entrante a las conexiones de vehículos para ir mejorando las rutas (Nguyen y Jung, 2021).

\section{MATERIALES Y MÉTODOS}

Esta investigación se realizó desde una perspectiva básicamente cuantitativa, desde un diseño descriptivo y no experimental. La recolección de datos se hizo con la ayuda del programa AutoCAD 2020 y la cartografía de la ciudad de Huaraz como instrumentos para el recojo de los datos. Este proceso de investigación inicia con el caso de estudio práctico y la búsqueda de información de los hoteles y su ubicación respectiva, así como las distancias correspondientes entre ellos y el lugar de parqueo. La población objetivo estuvo constituido por 85 hoteles, de los cuales se han tomado en cuenta 05 hoteles cuya ubicación relativamente diferenciada y dispersada dentro de la ciudad de Huaraz, zona urbana.

Una vez obtenida los datos, a través del programa AutoCAD 2020, y la elección de los 05 hoteles considerados en la muestra con el uso de la aplicación de la metaheurística colonia de hormigas, se realizó la elaboración de tablas de doble entrada, en primer lugar, para identificar las medidas en distancias de los 06 puntos-vértices, es decir un lugar de parqueo y cinco hoteles elegidos y luego para identificar el conjunto de rutas alternativas conformando el espacio de solución; asimismo, se hizo uso de la probabilidad y de números aleatorios en la aplicación de la metaheurística en las iteraciones de las hormigas artificiales y la actualización de la sustancia de feromona de las mismas.

\section{RESULTADOS}

\section{Tabla 1}

Distancias del punto-vértice lugar de parqueo a los hoteles (en metros)

\begin{tabular}{lcccccc}
\hline DEP. /HOTELES & $\begin{array}{c}\text { PUNTO 1: } \\
\text { C1 }\end{array}$ & $\begin{array}{c}\text { PUNTO 2: } \\
\text { C2 }\end{array}$ & $\begin{array}{c}\text { PUNTO 3: } \\
\text { C3 }\end{array}$ & $\begin{array}{r}\text { PUNTO 4: } \\
\text { C4 }\end{array}$ & $\begin{array}{r}\text { PUNTO 5: } \\
\text { C5 }\end{array}$ & $\begin{array}{c}\text { PUNTO 6: } \\
\text { C6 }\end{array}$ \\
\hline PUNTO 1: C1 & 0 & 403 & 456 & 1288 & 1348 & 543 \\
PUNTO 2: C2 & & 0 & 388 & 1691 & 1751 & 946 \\
PUNTO 3: C3 & & & 0 & 1388 & 1479 & 834 \\
PUNTO 4: C4 & & & & 0 & 435 & 928 \\
PUNTO 5: C5 & & & & & 0 & 752 \\
PUNTO 6: C6 & & & & & & 0 \\
\hline
\end{tabular}

El $5.89 \%$ de los hoteles fueron seleccionados como muestra para el estudio del problema de rutas en la ciudad de Huaraz. Las distancias en metros entre el conjunto de ellos y el lugar de parqueo indican medidas relativamente diferenciadas obtenidas a través del uso del programa de AutoCAD 2020. 
Tabla 2

Rutas encontradas por el ACO

\begin{tabular}{clc}
\hline $\mathrm{N}^{\circ}$ RUTA & RUTA & DISTANCIA(m) \\
\hline R001 & 1234561 & 3909 \\
R012 & 1246531 & 5709 \\
R026 & 1324651 & 5563 \\
R029 & 1326451 & 4501 \\
R030 & 1326541 & 4265
\end{tabular}

El 4,16\% del espacio de soluciones alternativas de rutas, a través de la aplicación metaheurística colonia de hormigas significa 05 rutas base, y de entre ellas se determina la ruta de recorrido mínimo.

\section{Tabla 3}

Iteraciones y sus probabilidades para las 5 hormigas

\begin{tabular}{ccccccc}
\hline $\begin{array}{c}\text { Hormiga } \\
\mathrm{k}\end{array}$ & $\begin{array}{c}1^{\circ} \\
\text { Probabilidad }\end{array}$ & $\begin{array}{c}2^{\circ} \\
\text { Probabilidad }\end{array}$ & $\begin{array}{c}3^{\circ} \\
\text { Probabilidad }\end{array}$ & $\begin{array}{c}4^{\circ} \\
\text { Probabilidad }\end{array}$ & $\begin{array}{c}5^{\circ} \\
\text { Probabilidad }\end{array}$ & $\begin{array}{c}6^{\circ} \\
\text { Probabilidad }\end{array}$ \\
\hline 1 & 0.310 & 0.724 & 0.195 & 0.180 & 1.000 & 1.000 \\
2 & 0.310 & 0.724 & 0.622 & 0.604 & 1.000 & 1.000 \\
3 & 0.310 & 0.724 & 0.622 & 0.396 & 1.000 & 1.000 \\
4 & 0.397 & 0.787 & 0.206 & 0.820 & 1.000 & 1.000 \\
5 & 0.397 & 0.041 & 0.166 & 0.551 & 1.000 & 1.000 \\
\hline
\end{tabular}

Se observa las probabilidades de las rutas uso de números aleatorios en la metaheurística encontradas por las hormigas artificiales con colonia de hormigas.

\section{Tabla 4}

Rutas actualizadas mediante la sustancia de feromona por el ACO

\begin{tabular}{ccc}
\hline $\mathrm{N}^{\circ}$ RUTA & $\begin{array}{c}\text { CONCENTRACIÓN DE } \\
\text { FEROMONA }\end{array}$ & DISTANCIA(m) \\
\hline R026 & 0.3085 & 5563 \\
R030 & 0.3103 & 4265 \\
R029 & 0.3100 & 4501 \\
R001 & 0.3110 & 3909 \\
R012 & 0.3082 & 5709 \\
\hline
\end{tabular}

Finalmente, se observa que la ruta de entender a un amplio número de problemas recorrido mínimo es la R001 con el mayor o de diversas actividades que desarrollan o valor de concentración de feromona 0.3110, generan precisamente recorridos o rutas para determinando el menor recorrido de $3909 \mathrm{~m}$. el cumplimiento de las mismas. Por ejemplo,

\section{DISCUSIÓN}

La propuesta sobre el problema de rutas en

la ciudad de Huaraz, conlleva a conocer el transporte público de pasajeros, el recojo de los residuos sólidos por parte del gobierno local, el servicio de movilidad escolar, el recojo y entrega de productos diversos, entre otros. El 
estudio y análisis de una situación problemática, en relación a la prestación de servicios de recojo de visitantes desde los hoteles en la ciudad de Huaraz, como centro receptor turístico, para ser transportados a los diversos destinos turísticos de la región, se hace complejo; no obstante, se asuma un marco de orden, considerando un punto 1: parqueo San Martín que es el lugar de salida y retorno de los vehículos de transporte turístico. Considerando que, los vehículos tienen que llegar puntualmente a recoger a los visitantes desde los hoteles sin tener demora, caso contrario significaría otra variable. Además, la incertidumbre en la información disponible sobre los visitantes a la ciudad de Huaraz origina desorden en la práctica de esta actividad y por ende a un malestar principalmente a quienes se les atiende, o en el tiempo de espera que los vehículos deberían tomar, mientras los visitantes en los hoteles demoran para abordar el transporte.

El planeamiento del transporte de recojo de los visitantes a la ciudad de Huaraz, involucra varias etapas secuenciales desde el caso simple de la aplicación del problema de rutas de vehículos determinando un conjunto de rutas que necesariamente involucran un lugar fijo y en otras varias y dispersadas con variables de al menos distancias, otras por ejemplo tiempo y costos; otro aspecto, es la capacidad de los vehículos de recojo, que permite el recojo de los visitantes desde los hoteles puede ser variables, en función del tipo de vehículo de transporte que se elija por ello preferentemente se mide la distancia al desarrollar el conjunto de rutas.

La solución de problemas normalmente está asociada inexorablemente a un proceso de búsqueda, salvo en problemas muy simples; la búsqueda de soluciones no puede realizarse en forma exhaustiva, por lo que las investigaciones han desarrollado una gran cantidad de métodos alternativos de búsqueda que encuentren soluciones aceptables y en muchos casos inclusive óptimas. Lo mismo se aplica para solucionar problemas de optimización para los cuales se han desarrollado métodos aproximativos, a veces involucrando cierta aleatoriedad. Por ello es imperativo y necesario hacer un planeamiento del transporte en este servicio, teniendo en cuenta que el servicio de recojo de visitantes es una actividad muy delicada y potencialmente en crecimiento.

Las hormigas intercambian información de manera indirecta depositando feromonas en su recorrido, solamente una hormiga ubicada cerca de donde las feromonas fueron depositadas puede ser tomada en cuenta para los propósitos de un diseño de ruta. El mecanismo para resolver un problema demasiado complejo para ser abordado por hormigas artificiales solamente es un buen ejemplo de un sistema auto-organizado. Este sistema está basado en la retroalimentación positiva (el depósito de feromonas atrae otras hormigas y estas fortalecerán dicha retroalimentación) y la retroalimentación negativa (disipación de la ruta por evaporación). Teóricamente se asume que, si la cantidad de feromonas fue la misma en todas las rutas durante todo el tiempo, ninguna ruta fue elegida. Sin embargo, debido a la retroalimentación, una ligera variación en una arista amplificará y entonces se permitirá elegir una ruta. El algoritmo se moverá de un estado inestable en el que ninguna arista es más fuerte que otra, a un estado estable donde una ruta está compuesta por las aristas más fuertes.

La filosofía básica del algoritmo implica el movimiento de una colonia de hormigas a través de los diferentes estados del problema influenciado por dos políticas de decisión a nivel local, rutas y atracción. De esta manera, cada hormiga artificial incrementalmente construye una solución del problema. Cuando una hormiga completa una solución, o durante la fase de construcción, las hormigas artificiales evalúan la solución y modifican el valor de la ruta sobre las componentes utilizadas en la solución. Esta información de feromonas dirigirá la búsqueda de futuras hormigas. La evaporación del rastro reduce todos los valores de los rastros evitando la posibilidad de caer en óptimos locales.

Los estudios de transporte en ruteos con ayuda de herramientas científicas aún son muy incipientes en el país, por ello es muy necesario puedan ser abordados y ser extendidos en diversos escenarios de investigación, según Garrido H. (2010). 
Es ese sentido, la pertinencia del presente estudio cobra el interés en una de las principales actividades en la región de Ancash, teniendo en cuenta que la actividad turística constituye un ámbito muy complejo y delicado en razón de que está orientado a las personas.

El ACO multinivel en sus diversas aplicaciones resuelve problemas de planeamiento de transportes en actividades forestales. Los parámetros de ACO son automáticamente configurados al evaluar un parámetro de dominio combinado a través de varios niveles (Lin, Contreras, Dai y Zhang, 2016). Así como pueden ser las aplicaciones del ACO orientado a otras diversas actividades, siempre será muy útil y necesario en cuanto se refiera a las actividades de transporte.

El ACO siendo una metaheurística de aproximación que converge a las soluciones en un tiempo razonable en un determinado problema, puede ayudar también a encontrar buenas soluciones a algunos problemas que podrían ser incluso muy restringidos (Baeza, Ihle y Ortiz, 2017). Las medidas de distancias en los problemas de la optimización combinatoria, hace referencia que no solo son longitudes, son también tiempos o costos, esta riqueza de medidas conlleva a la posibilidad de atender variadas problemáticas, como por ejemplo los servicios turísticos, y la gran complejidad que conlleva atenderlos.

T Srinivas Rao (2018), concluyó que el ACO contribuye a las soluciones de la necesidad de cadenas de abastecimiento en administración logística siendo una buena alternativa a través del uso de los VRP específicamente en las ciudades, determinando bajos costos y calculando las rutas más cortas. En este sentido, significa argumentos que la metaheurística colonia de hormigas está plenamente validada por sus resultados investigativos.

El mTSP Problema del Agente Viajero múltiple es aplicado a trabajos en equipos con una misión utilizando una estrategia max-min para trabajar juntos en la búsqueda de la solución óptima, según Li-Chih y Tai-Wen (2019). Esto da pie, a que la problemática de los servicios turísticos aun siendo una actividad muy compleja de cumplir, permite dar margen a atender diversas nuevas atenciones, posiblemente con detalles y especificaciones.

Según Li-Chih Lu (2019), "el VRP se simplifica a través de las soluciones del tipo mTSP, ACO y propiamente a las hormigas que forman equipos simulando misiones y combinando estrategias trabajando juntas para optimizar la solución, teniendo como meta reducir la distancia total"; esto significa los propósitos del algoritmo colonia de hormigas siempre son determinar soluciones óptimas o eficientes, o en todo caso suficientes.

El ACO integra también rutas de tráfico dinámico para la conexión de vehículos donde son modelados como una hormiga, concluye Nguyen y Jung (2021). Esto es el mecanismo natural y trascendente del algoritmo colonia de hormigas, así como la naturaleza de la emisión de la sustancia de feromona, que según su incidencia determinará los caminos que permitan determinar el recorrido final con la mínima distancia.

Una de las restricciones del tránsito de los vehículos pesados, hace que la mayoría de las ciudades hayan empezado a implementar centros receptores para reducir los impactos negativos ambientales con ayuda de modelos matemáticos con el objetivo de minimizar los costos totales de transporte incluyendo costos de rutas, costos de arreglos de vehículos, costos de emisión y de impuestos zonas de entrada, concluye Juvvala y Sarmah (2021). En relación a lo indicado, precisamente en la actividad turística de la región de Ancash, la ciudad de Huaraz, constituye ese centro receptor, pues a partir de esta ciudad se generan las salidas de la prestación de servicios a los destinos turísticos de la región de Ancash.

En los problemas de optimización combinatoria, conforme aumenta el tamaño de un problema por el número de puntos-vértice seleccionados, las combinaciones de entre ellas crece de manera exponencial, y que en cierto momento se imposibilita lograr un desarrollo manual, y las herramientas informáticas constituyen la ayuda 
indispensable para obtener resultados.

Finalmente, en relación al número de puntosvértices tomados en número de 06 , la determinación y análisis de la disposición de 720 rutas en el caso de estudio práctico tienen una significancia relativamente alta, y que en el marco de la optimización combinatoria resulta muy laborioso evaluar cada una de ellas; sin embargo, con conocimiento de la naturaleza del problema del agente viajero, los ruteos ocurren eligiendo o determinando un punto-vértice, lo que disminuye el espacio de soluciones alternativas a un número de 120 rutas; a ventaja de ello, aún resulta arduo. Realizando las combinaciones de ruteos correspondientes, se generan coincidencias de valores totales en los ruteos, haciendo que 76 rutas resulten ser iguales en su valoración y solo 46 de ellas tengan valores diferentes.

\section{CONCLUSIONES}

El $5.89 \%$ de los hoteles equivalente a 05 de ellos fueron considerados como la muestra de investigación, son del conjunto de hoteles que cuentan con muchos años desde su instalación en la ciudad de Huaraz, y que estratégicamente se encuentran bien ubicados para el estudio y aplicación de la metaheurística colonia de hormigas.

El 4,16\% del espacio de soluciones alternativas de rutas equivalente a 05 rutas, son producto de la aplicación de la metaheurística colonia de hormigas, y que aún con la aplicación la misma, los resultados no son siempre todos los menores en cuanto a su longitud.

El uso de los números aleatorios después de las probabilidades obtenidas en el proceso de la aplicación de la metaheurística colonia de hormigas, no garantiza que coincidan con la mejor de las probabilidades resultantes.

La mayor concentración de la sustancia de feromona y hormigas artificiales juega un rol determinante en la mejora de los caminos que generan la ruta y al final contribuyen a la elección de la ruta de recorrido mínimo, en el caso de la aplicación fue la R001 con 3909 m.

\section{REFERENCIAS BIBLIOGRÁFICAS}

Baeza, D., Ihle, C., Ortiz, J. (2017). A comparison between $\mathrm{ACO}$ and Dijkstra algorithms for optimal ore concentrate pipeline routing. Advanced Laboratory for Geostatistical (ALGES), University of Chile, Santiago, Chile. https://doi.org/10.1016/j. jclepro.2016.12.084

Garrido, A. (2010). Planeamiento del transporte de clientes turísticos en Huaraz bajo un enfoque multicriterio. Escuela de Postgrado. Universidad Nacional Santiago Antúnez de Mayolo, 72-73. Disponible en: http://repositorio.unasam.edu.pe/handle/ UNASAM/884

Juvvala, R., \& Sarmah, S. P. (2021). Evaluation of policy options supporting electric vehicles in city logistics: a case study. Sustainable Cities and Society, 74, 103209. https://doi.org/10.1016/j.scs.2021.103209

Li-Chih L., Tai-Wen Y. (2019). Missionoriented ant-team ACO for min-max MTSP. Department of Computer Science and Engineering, Tatung University, $\mathrm{N}^{\circ} 40$. Taipei, Taiwan. https://doi.org/10.1016/j. asoc. 2018.11 .048

Lin, P., Contreras, M., Dai, R., Zhang, J. (2016). A multinivel ACO approach for solving forest transportation planning problems with environmental constraints. Department of Mathematics, Statistic and Computer Science, University of WisconsinStout, Menomonie, USA. DOI: 10.1016/j. swevo.2016.01.003

Nguyen, T., Jung, J. (2021). Ant colony optimization-based traffic routing with intersection negotiation for connected vehicles. Department of Computer Engineering, Chung-Ang University, Dongjak, Republico of Korea. https://doi. org/10.1016/j.asoc.2021.107828

T Srinivas Rao (2018). An Evaluation of ACO and GA TSP in a Supply Chain Network. School of Engineering, Amrita Vishwavidyapeetham, India. matpr.2018.10.338 https://doi.org/10.1016/j. 